\title{
Correspondence
}

\section{Cell-based therapies for coronavirus disease 2019: proper clinical investigations are essential}

\author{
Maroun Khoury ${ }^{1,2}$, Patricia R.M. Rocco ${ }^{3,4}$, Donald G. Phinney ${ }^{5}$, Mauro Krampera ${ }^{6}$, \\ Ivan Martin $^{7}$, Sowmya Viswanathan ${ }^{8,9}$, Jan A. Nolta ${ }^{10}$, Katarina LeBlanc ${ }^{11}$, Jacques Galipeau ${ }^{12}$, \\ Daniel J. Weiss ${ }^{13, *}$ \\ ${ }^{1}$ Laboratory of Nano-Regenerative Medicine, Faculty of Medicine, Universidad de los Andes, Santiago, Chile \\ ${ }^{2}$ Cells for Cells and Consorcio Regenero, Chilean Consortium for Regenerative Medicine, Santiago, Chile \\ ${ }^{3}$ Laboratory of Pulmonary Investigation, Carlos Chagas Filho Institute of Biophysics, Federal University of Rio de Janeiro, Rio de Janeiro, Brazil \\ ${ }^{4}$ National Institute of Science and Technology for Regenerative Medicine, Rio de Janeiro, Brazil \\ ${ }^{5}$ Department of Molecular Medicine, The Scripps Research Institute, Jupiter, Florida, USA \\ ${ }^{6}$ Section of Hematology, Department of Medicine, University of Verona, Verona, Italy \\ ${ }^{7}$ Department of Biomedicine, University Hospital Basel, University of Basel, Basel, Switzerland \\ ${ }^{8}$ Krembil Research Institute, University Health Network, Toronto, Canada \\ ${ }^{9}$ Institute of Biomaterials and Biomedical Engineering and Department of Medicine, University of Toronto, Toronto, Canada \\ ${ }^{10}$ Institute for Regenerative Cures, University of California Davis Health, Sacramento, California, USA \\ ${ }^{11}$ Department of Laboratory Medicine, Karolinska Institutet, Cell Therapy and Allogeneic Stem Cell Transplant, Patient Area Cell Therapies and Allogeneic Stem Cell \\ Transplantation, Karolinska University Hospital, Stockholm, Sweden \\ ${ }^{12}$ Department of Medicine, University of Wisconsin Carbone Comprehensive Cancer Center, University of Wisconsin-Madison, Madison, Wisconsin, USA \\ ${ }^{13}$ Department of Medicine, University of Vermont, Burlington, Vermont, USA
}

\section{A R T I C L E I N F O}

\section{Article History:}

Received 3 April 2020

Accepted 13 April 2020

\section{Key Words:}

acute respiratory distress syndrome

cell therapy

coronavirus

COVID-19

mesenchymal stromal cells

MSCs

\begin{abstract}
A B S T R A C T
The serious consequences of the global coronavirus disease 2019 (COVID-19) pandemic have prompted a rapid global response to develop effective therapies that can lessen disease severity in infected patients. Cellbased approaches, primarily using mesenchymal stromal cells (MSCs), have demonstrated a strong safety profile and possible efficacy in patients with acute respiratory distress syndrome (ARDS), but whether these therapies are effective for treating respiratory virus-induced ARDS is unknown. According to the World Health Organization International Clinical Trials Registry Platform and the National Institutes of Health ClinicalTrials.gov databases, 27 clinical investigations of MSC-based cell therapy approaches have begun in China since the onset of the COVID-19 outbreak, with a growing number of academic and industry trials elsewhere as well. Several recent published reports have suggested potential efficacy; however, the available data presented are either anecdotal or from incomplete, poorly controlled investigations. Therefore, although there may be a potential role for MSCs and other cell-based therapies in treatment of COVID-19, these need to be investigated in a rationally designed, controlled approach if safety and efficacy are to be demonstrated accurately. The authors urge that the field proceed by finding a balance between swift experimentation and communication of results and scientifically coherent generation and analysis of clinical data.
\end{abstract}

(c) 2020 Published by Elsevier Inc. on behalf of International Society for Cell \& Gene Therapy.

\section{Introduction}

The number of patients infected as a result of the ongoing coronavirus disease 2019 (COVID-19) pandemic continues to increase rapidly worldwide. Mounting data demonstrate that infection with severe acute respiratory syndrome coronavirus 2 , the viral causative agent of COVID-19, can result in significant respiratory morbidity and mortality [1]. Severe pneumonia and acute respiratory distress

\footnotetext{
* Correspondence: Daniel J. Weiss, MD, PhD, 226 Health Sciences Research Facility, Department of Medicine, University of Vermont, Burlington, Vermont, USA.

E-mail address: daniel.weiss@med.uvm.edu (D.J. Weiss).
}

syndrome (ARDS) have been described in $14 \%$ of reported cases, and the overall fatality rate is currently estimated at $2 \%$ [2]. However, these numbers continue to evolve as the pandemic spreads and region-specific differences in rates of infection and patient outcomes emerge. Current therapeutic approaches include aggressive standard supportive care and treatment of any other co-infections. Anti-viral and immune-based approaches, such as anti-IL- 6 or anti-IL- 1 therapy, are also under investigation, but their safety and potential efficacy have yet to be determined.

A growing number of clinical investigations of cell-based therapies have been initiated in China for COVID-19 respiratory disease, 
encompassing a wide range of approaches and targeted patient groups. Although these include several cell types, most are directed at the use of mesenchymal stromal cells (MSCs) or MSC-derived conditioned media or extracellular vesicles. In a parallel article, the authors consider the rationale for the use of MSC-based therapies and their potential mode of action in respiratory virus infections and ARDS, review pre-clinical data from models of respiratory virus infections and survey the ongoing clinical studies of MSC administration taking place in China [3]. In the present review, two recently published clinical investigations of systemic MSC administration in patients with either COVID-19 or avian influenza A(H7N9) are considered $[4,5]$.

\section{Clinical investigations of MSC administration in patients with coronavirus- or other respiratory virus-induced lung injury}

In a recently published single-center, open-label pilot investigation conducted at YouAn Hospital in Beijing, MSCs were administered to seven patients with COVID-19 pneumonia with differing degrees of severity, including one critically ill patient requiring intensive care unit care [4]. MSCs were given as a single intravenous administration at a dose of $1 \times 10^{6}$ cells $/ \mathrm{kg}$ body weight in a total volume of $100 \mathrm{~mL}$ saline at various time points after initial symptomatic presentation. As described in the report, "The window period for cell transplantation was defined as the time when symptoms were getting worse even as expectant treatments were being conducted." The MSCs utilized were certified by the National Institutes for Food and Drug Control of China, but no information was provided regarding tissue source or mode of manufacturing. Notably, the MSCs were assessed by RNA sequencing for expression of ACE2 or TMPRSS2 prior to administration, and each was found to be minimally expressed (1/12 500 cells and 7/12500 cells, respectively). Based on this result, the MSCs were assumed to be free of COVID-19 infection. However, RNA sequencing results were not validated for gene (quantitative realtime polymerase chain reaction) or protein expression (western blot) to confirm ACE2 or TMPRSS2 expression levels and therefore did not rule out the possibility of false-negative results.

The seven treated patients were categorized as critically severe $(\mathrm{n}=1)$, severe $(\mathrm{n}=4)$ and "common type" (assumed to be mild to moderate disease; $\mathrm{n}=2$ ). Three patients classified as severe received placebo (vehicle) for comparison. Patients were followed for 14 days after MSC or placebo administration. The primary endpoint measures included (i) primary safety data (infusional toxicity, subsequent allergic reactions, secondary infections and life-threatening adverse events), (ii) clinical outcomes and (iii) levels of circulating inflammatory mediators, including levels of pro- and anti-inflammatory cytokines and C-reactive protein. Secondary assessments included (i) clinical symptoms, (ii) radiographic (chest computed tomography) changes and (iii) circulating lymphocyte numbers and populations. Standard supportive therapies and anti-viral medications were continued as per routine clinical care. As described by the authors, no infusional toxicities, allergic reactions, secondary infections or severe attributable adverse events were observed over the 14-day period. This is consistent with the general experience with single intravenous administration of MSCs in a range of clinical settings, including a phase 2a clinical study in ARDS [6]. All patients, including the one categorized as critically severe, demonstrated clinical improvements within 2-4 days after MSC administration, including resolution of clinical symptoms (fever, cough, elevated respiratory rate) and improvements in oxygen saturation. However, although detailed information is provided for the critically severe patient, there is a lack of corresponding information for the other six patients. The three placebo patients are only briefly described as doing poorly, with one described as having died, one described as having ARDS and one categorized as severe. Detailed information about their clinical course and concomitant therapy is also lacking.
Extensive analyses of circulating pro- and anti-inflammatory mediators and lymphocyte numbers and populations are presented in detail for the critically severe patient. These analyses demonstrated an initial increase in C-reactive protein 1 day after MSC administration and a subsequent decline over the 14-day observational period. Six days after MSC administration, the levels of circulating CXCR3+ CD4, CD8 and natural killer cells decreased. These limited data suggest that levels of $\mathrm{T}$ regulatory cells and CXCR3-dendritic cells increased in the severe and critically severe patients but not in the "common type" patients or in the placebo controls. Data presented on three patients demonstrated a statistically significant increase in circulating IL-10 and a trend toward increased vascular endothelial growth factor and IP-10 levels. A statistically significant decrease in tumor necrosis factor alpha was observed in the same three patients. However, it was not clear what the timing of measurements was after MSC (or placebo) administration, nor which patients were targeted for these measurements, including whether any placebo controls were included. Similarly, limited data on circulating viral titers were presented for the critically severe patient, demonstrating that titers were persistently positive 6 days after MSC administration, becoming negative by 13 days. However, no quantitative data were presented, and no other information was presented for any of the other patients.

The authors appreciate that under the current pandemic conditions it may be difficult to recruit patients for placebo controlled trials and that patient randomization is complicated by the fact that multiple agents are being utilized and/or investigated. They also appreciate the need for rapid publication of results from pilot clinical trials in COVID19 patients. In light of these limitations, interpreting the impact of the clinical data reported by Leng et al. [4] requires additional information on comorbidities, the clinical course of each patient and their subsequent outcomes. Importantly, because of the limited amount of information on the placebo control patients, it is difficult to determine whether MSC administration truly affected the clinical course of the disease or if the clinical outcomes reflected variation in the natural course of infection in different patients. Moreover, although including detailed assessments of circulating inflammatory mediators and lymphocyte populations is necessary to understand the mode of action of the administered MSCs, as demonstrated in other recent investigations of ARDS and chronic obstructive pulmonary disease patients [7-9], these data were limited and in some cases unclear in the analyzed case report. Consequently, although the authors speculate about the mechanism of MSC action in COVID-19 patients and the effects on the immune system, definitive results will require large-scale, randomized controlled trials to delineate mode(s) of action, which may be disease-specific. Importantly, although the authors did not discuss potential patient targeting for MSC administration, it is important to determine if the approach should be further investigated in only critically severe and/or severe patients or in the broader range of clinical presentations of COVID-19 respiratory infection.

A second recent study evaluated MSC administration in patients with influenza $\mathrm{A}(\mathrm{H} 7 \mathrm{~N} 9)$ infection [5]. This was also a single-center, open-label investigation, conducted at Zheijang University during the 2013-2014 H7N9 outbreak. In this study, 17 critically ill patients with virus-induced ARDS received multiple intravenous administrations of menstrual blood-derived MSCs obtained from a single healthy donor, and their outcomes were compared with 44 comparably critical patients receiving standard anti-viral and supportive therapies. Among the treated patients, three were described as receiving three separate infusions during "early-stage" infection, six as receiving three infusions during "late-stage infection" and eight as receiving four infusions during "late-stage infection." The MSCs were suspended in Plasma-Lyte A at a concentration of $1 \times 10^{6}$ cells/infusion, but the final volume was unclear. According to the report, "[T] he total usage of MSC was $100 \mathrm{~mL}$ for each patient in the experimental group." Furthermore, no information was provided about the timing between infusions or whether the control patients received 
vehicle infusions. The MSC-treated and control patients were fairly well matched for comorbidities, degree of multiorgan failure and use of other supportive therapies, except for a higher incidence of shock in the MSC-treated group $(P<0.03)$

No apparent infusional toxicities or serious adverse events were noted. Three patients in the MSC-treated group died ( $82.4 \%$ survival) versus 24 in the control group (45.5\% survival). However, no details on the deaths, including cause and timing related to either infusion or overall clinical course, were provided. Moreover, no other outcome measures now considered standard for evaluating new potential therapies in intubated, mechanically ventilated patients-such as ventilator-free days, length of intensive care unit stay or length of hospital stay-were reported. Limited long-term follow-up data were presented for a small number of treated patients and appeared to indicate no difference in long-term sequelae observed in ARDS survivors compared with those reported in the literature. Baseline laboratory data, including complete blood count and measures of renal, liver, cardiac and coagulation functions, were comparable, except for a higher circulating procalcitonin level in the control group (perhaps suggestive of secondary or co-occurring bacterial superinfection). However, no information on other infections was provided. There were many differences in laboratory values between treated and control patients at discharge; however, no information as to the definition of discharge was provided, nor was any other relevant information about clinical course reported. Based on these findings, the authors concluded that MSC administration is a viable approach for treating ARDS resulting from influenza $A(H 7 N 9)$ infection and could be potentially applicable for use in coronavirus-related respiratory infections.

These two studies, although suggestive, highlight a number of critical parameters that should be rigorously evaluated when assessing the potential use of MSCs in coronavirus and other viral respiratory infections. These include but are not limited to source of MSCs, dose and dosing strategies (including the number and timing of administrations). It stands to reason that the optimal criteria for the selection of these metrics are determined by the target indication, not by cell availability or ease of cell isolation. Furthermore, these choices need to be backed by robust experimental data. An additional factor that was not clarified in either of these trials and that may be fundamental to the success of MSC-based cell therapies for any disease indication is the use of fresh versus cryopreserved and ready-toinject versus cell culture-conditioned cells [9]. These studies also highlight issues regarding the conduct of clinical trials for respiratory diseases, including those in critically ill patients. Full information about inclusion and exclusion criteria, clinical course, comorbidities, co-infections and laboratory tests, including investigative mechanistic evaluations, must be provided in a comprehensive manner. This information should also include a robust description of statistical measures used to assess cohort sample sizes and to evaluate differences in outcome measures between treatment groups. Although this level of rigor in reporting may be difficult to achieve under pandemic conditions, all authors and managing editors should aim to publish comprehensive results to inform future clinical trials and patient treatment options.

\section{Clinical-stage industrial MSC pharmaceutical providers and COVID-19}

In addition to investigations ongoing in China and various academic institutions in Europe, North America and elsewhere, several clinical-stage biopharmaceutical companies, including Athersys, Inc., Mesoblast, Inc. and Pluristem, Inc., are planning clinical trials to test their respective MSC-like products on COVID-19-associated ARDS. These trials span the gamut from compassionate use/hospital exemption to phase 3 studies, and the respective platforms employ MSCs from different sources, such as bone marrow (Athersys, Inc. and
Mesoblast, Inc.) and placenta (Pluristem, Inc.), and also employ different routes of delivery, including intravenous (Athersys, Inc., Mesoblast, Inc.) and extravascular (Pluristem, Inc.). Industries may be well positioned to conduct large, informative clinical trials in a timely fashion and acquire the necessary data to obtain market approval for their respective platforms by national regulatory bodies. However, these trials must also be conducted under appropriate trial design, regulatory oversight and transparency of reporting results. The companies just listed have all announced ongoing conversations with national regulators to launch trials and begin treatment of the first subjects in a short time span [10-12].

\section{Ethical issues and guidelines when considering cell-based approaches for COVID-19 respiratory virus infections}

As governmental organizations, health care providers and clinical investigators around the world attempt to navigate these unprecedented times, they need to lead by identifying and selecting only the most pertinent evidence for clinical testing and authorization. These pressing actions still need to occur under appropriate ethical and scientific guidelines and undergo an appropriate, scientifically rigorous approval process overseen by duly empowered agencies. The ethical guidelines provided by the World Health Organization are worthy baselines applicable to the many cell-based clinical trials that are being planned as a response to the COVID-19 public health emergency [13].

A highlight of this moral compass includes the registration of all clinical trials, just as would be required in non-outbreak situations. It is also essential that the expected risks be reasonable in relation to anticipated benefits. In addition, other factors can challenge the trial's sponsors to obtain informed consent, as prospective participants might be in intensive care. One suggested solution is the appointment of proxy decision makers that can sign consent on their behalf. This is an ethical issue that is approached differently in different countries. The US Food and Drug Administration has issued guidance to provide general considerations to assist sponsors in assuring the safety of trial participants, maintaining compliance with good clinical practice and minimizing risks to trial integrity during the COVID-19 pandemic [14]. These guidelines are intended for ongoing trials that are not related to the treatment of COVID-19 patients. However, many of the assessments can also be applicable to planned COVID-19 trials. New logistics are proposed to cope with enforced confinement; notably, as trial participants may not be able to come to the investigational site for specified visits, sponsors are allowed to evaluate whether alternative methods can be implemented for remote safety assessments. Also, changes to the protocol to minimize or eliminate immediate hazards may be implemented without institutional review board approval but must still be reported afterward.

More directly, the US Food and Drug Administration has created a special emergency program-the Coronavirus Treatment Acceleration Program-for possible therapies [15]. The program is meant to fasttrack new treatments to patients, provide immediate triage and ensure response within a day of request receipt. Interactions are prioritized on the basis of each product's stage of development, with protocol review of prioritized products within $24 \mathrm{~h}$ of submission and expedited quality assessments for products to treat COVID-19 patients and to transfer manufacturing to alternative or new sites to avoid supply disruption. Other decisions include the redeployment of medical, operations, policy and regulatory staff to review teams dedicated to COVID-19 therapies as well as involvement of senior management in review of submissions. Most importantly, the program provides resources to health care providers and researchers to help them submit emergency requests to use investigational products in patients with COVID-19. All of these are applicable to cell-based therapy investigations. 


\section{Discussion}

MSCs have been administered to large numbers of patients in various clinical contexts without evidence of significant safety-related issues [16], but less data are available for critically ill patients. Importantly, data from a phase 2a trial in ARDS due to other etiologies reported no significant infusional toxicities or other safety issues other than the death of one patient $24 \mathrm{~h}$ after MSC infusion, which was judged to be unrelated to cell administration [6]. With respect to clinical efficacy, results to date are ambiguous. For example, the phase 2a ARDS START trial reported a trend toward higher mortality in the MSC versus placebo arm that was attributed to patient randomization [6], and results from a phase 1/2 trial of MSC administration in ARDS sponsored by Athersys, Inc. are awaiting publication. One significant unresolved issue in this respect is which COVID-19 patient population to target. Critically ill patients with ARDS requiring supportive measures, including intubation and mechanical ventilation, are a logical population. Arguments can also be made for severely infected patients with currently recognized risk factors, such as advanced age, cardiovascular disease or hematologic malignancies requiring immunotherapy or chemotherapy. Whether patients with moderate or mild disease should be enrolled in clinical investigations remains less clear [3]. Another significant issue is the evolving understanding of the pathogenesis of ARDS in COVID-19 patients, how this may differ from other etiologies and the corresponding effects on potential cell-based therapies.

It is also imperative that clinical investigations be conducted in a transparent manner, according to established precedents for clinical research of new therapies in critical illness [17]. The authors also advocate rapid and full data sharing, believing that every researcher who engages in generation of information related to a public health emergency or acute public health event with the potential to progress to an emergency has a fundamental moral obligation to share preliminary results once they are adequately quality controlled for release. Given the rapid spread of COVID-19 and the increasing numbers of cell-based therapy investigations, a coordinating center to expedite congruent trial design and appropriate data dissemination would be of significant benefit.

The authors must also take a strong stance against the rogue stem cell clinic industry, which has already begun to offer unproven therapies for COVID-19. The International Society for Cellular and Gene Therapies [18], International Society for Stem Cell Research [19], Alliance for Regenerative Medicine [20] and other organizations have aligned their stances against this predatory behavior, but more regulatory oversight and action are needed. Only then can a rational evidence-based platform for potential therapeutic use of cell-based therapies be developed

\section{Funding}

No funding was received.

\section{Declaration of Competing Interest}

MK is the chief scientific officer of Cells for Cells.

\section{Author Contributions}

Conception and design of the study: MK (Khoury), DJW. Analysis and interpretation of data: All authors. Drafting or revising the manuscript: All authors. All authors have approved the final article.

\section{References}

[1] De Wit E, Van Doremalen N, Falzarano D, Munster VJ. SARS and MERS: Recent insights into emerging coronaviruses. Nat Rev Microbiol 2016;14:523-34.

[2] Zhu N, Zhang D, Wang W, Li X, Yang B, Song J, et al. A Novel Coronavirus from Patients with Pneumonia in China, 2019. N Engl J Med 2020;382:727-33.

[3] Khoury M, Cuenca J, Cruz FF, Figueroa FE, Rocco PRM, Weiss M, et al. Current Status of Cell-Based Therapies for Respiratory Virus Infections: Applicability to COVID-19. Eur Respir J 2020:55:2000858.

[4] Leng Z, Zhu R, Hou W, Feng Y, Yang Y, Han Q et al. Transplantation of ACE2- Mesenchymal Stem Cells Improves the Outcome of Patients with COVID-19 Pneumonia. Aging Dis 2020;11:216-28.

[5] Chen J, Hu C, Chen L, Tang L, Zhu Y, Xu X, et al. Clinical study of mesenchymal stem cell treating acute respiratory distress syndrome induced by epidemic Influenza A (H7N9) infection, a hint for COVID-19 treatment. Engineering (Beijing) 2020. https://doi.org/10.1016/j.eng.2020.02.006.

[6] Matthay MA, Calfee CS, Zhuo H, Thompson BT, Wilson JG, Levitt JE, et al. Treatment with allogeneic mesenchymal stromal cells for moderate to severe acute respiratory distress syndrome (START study): a randomised phase 2a safety trial. Lancet Respir Med 2019:7:154-62.

[7] Simonson OE, Mougiakakos D, Heldring N, Bassi G, Johansson HJ, Dalén M, et al. In Vivo Effects of Mesenchymal Stromal Cells in Two Patients With Severe Acute Respiratory Distress Syndrome. Stem Cells Transl Med 2015;4:1199-213.

[8] Armitage J, Tan DBA, Troedson R, Young P, Lam KV, Shaw K, et al. Mesenchymal stromal cell infusion modulates systemic immunological responses in stable COPD patients: a phase I pilot study. Eur Respir J 2018;51:1702369.

[9] Galipeau J, Sensébé L. Mesenchymal Stromal Cells: Clinical Challenges and Therapeutic Opportunities. Cell Stem Cell 2018;22:824-33.

[10] Athersys Stem Cell Therapy Targets Top Cause of COVID-19 Deaths n.d. https:// www.genengnews.com/gen-edge/athersys-stem-cell-therapy-targets-top-causeof-covid-19-deaths/. (Accessed April 2, 2020).

[11] Israeli company uses placenta cells to treat critical COVID-19 patients - The Jerusalem Post n.d. https://www.jpost.com/HEALTH-SCIENCE/Israels-company-usesplacenta-cells-to-treat-critical-COVID-19-patients-622960. (Accessed April 2, 2020).

[12] Mesoblast To Evaluate Anti-Inflammatory Cell Therapy Remestemcel-L For Treatment Of COVID-19 Lung Disease | BioSpace n.d. https://www.biospace.com/article/releases/mesoblast-to-evaluate-anti-inflammatory-cell-therapy-remestemcel-1-for-treatment-of-covid-19-lung-disease/. (Accessed April 2, 2020).

[13] Organisation WH. Guidance for managing ethical issues in infectious disease outbreaks. World Heal Organ 2016: 62

[14] FDA. FDA Guidance on Conduct of Clinical Trials of Medical Products during COVID-19 Pandemic Guidance for Industry, Investigators, and Institutional Review Boards. 2020.

[15] FDA. Coronavirus Treatment Acceleration Program (CTAP) | FDA n.d. https:// www.fda.gov/drugs/coronavirus-covid-19-drugs/coronavirus-treatment-acceleration-program-ctap. (Accessed April 1, 2020).

[16] Lalu MM, McIntyre L, Pugliese C, Fergusson D, Winston BW, Marshall JC, et al. Safety of cell therapy with mesenchymal stromal cells (SafeCell): a systematic review and meta-analysis of clinical trials. PLoS One 2012;10:e47559.

[17] Keusch GT, McAdam K, Cuff PA, Mancher M, Busta ER. Integrating clinical research into epidemic response: the Ebola experience. National Academies Press; 2017. https://doi.org/10.17226/24739.

[18] ISCT Releases Statement on Unproven Stem Cell Treatments for COVID-19 ISCT n.d. https://isctglobal.org/news/494824/ISCT-Releases-Statement-onUnproven-Stem-Cell-Treatments-for-COVID-19.htm. (Accessed March 20, 2020).

[19] ISSCR Statement Regarding the Marketing of Unproven Stem Cell Treatments for COVID-19 n.d. https://www.isscr.org/news-publicationsss/isscr-news-articles/ article-listing/2020/03/06/isscr-statement-regarding-the-marketing-ofunproven-stem-cell-treatments-for-covid-19. (Accessed March 18, 2020).

[20] Not All Stem Cell Treatments Are Equal-Alliance for Regenerative Medicinen.d. https://alliancerm.org/bioethics/stem-cell-clinical-trials/. (Accessed April 2 , 2020). 